\title{
DOR CRÔNICA E PREJUÍZOS NAS ATIVIDADES COTIDIANAS DE IDOSOS
}

\author{
Luciano Ramos de Lima ${ }^{1}$ \\ Isabella Cristina Severina ${ }^{2}$ \\ Ana Catarina Guimarães Silva ${ }^{3}$ \\ Silvana Schwerz Funghetto ${ }^{4}$ \\ Tania Cristina Morais Santa Barbara Rehem ${ }^{5}$ \\ Walterlânia Silva Santos ${ }^{6}$ \\ Cris Renta Grou Volpe ${ }^{7}$ \\ Marina Morato Stival ${ }^{8}$ \\ Mani Indiana Funez ${ }^{9}$ \\ Recebido em: 08 fev. 2018 \\ Aceito em: 19 nov. 2018
}

RESUMO: Objetivo: Analisar os principais prejuízos associados a dor crônica de um grupo de idosos. Método: Estudo transversal, descritivo de abordagem quantitativa. A amostra de 268 idosos, os instrumentos adotados: sócio demográfico, clínico, para avaliar a dor crônica (Escala Visual Analógica/EVA e diagrama corporal) e outro relacionado aos prejuízos no cotidiano. Resultados: A maioria $(67,5 \%)$ foi do sexo feminino com idade média de 68,9 anos e a dor foi caracteriza como intensa. Os principais prejuízos foram nas Atividades de Vida Diária (AVD) e na relação com outras pessoas $(p=\leq 0,02)$ associados a maior intensidade de dor. O principal local de dor referido foi no tronco e acomete 39,9\% durante a noite. Conclusão: Os principais prejuízos associado a dor foi nas AVD e na relação com outras pessoas. A dor que acomete os idosos é caracterizada como intensa e aparece no período da noite.

Palavras-chave: Idoso. Dor crônica. Avaliação em enfermagem. Serviços de saúde para idosos. Medição da Dor. Cuidados. Enfermagem primária.

\section{CHRONIC PAIN AND LOSSES IN THE DAILY ACTIVITIES OF THE ELDERLY}

ABSTRACT: Objective: To analyze the main damages associated with chronic pain in a group of elderly people. Method: Cross-sectional study, descriptive of quantitative approach. The sample of 268 elderly people, the instruments adopted: demographic,

\footnotetext{
1 Professor Assistente do curso de Enfermagem da Universidade de Brasília/Campus Ceilândia, Doutorando PPCTS/UNB, Mestre FEN/UFG, E-mail: ramos\|@unb.br.

2 Enfermeira graduada pela Universidade de Brasília/Campus Ceilândia, Bolsista CNPQ de Iniciação científica, E-mail: isabella.c.severina@hotmail.com.

3 Enfermeira graduada pela Universidade de Brasília/Campus Ceilândia, Bolsista CNPQ de Iniciação científica, E-mail: anacatarina.enf@gmail.com.

4 Professora Adjunta do curso de Enfermagem da Universidade de Brasília/Campus Ceilândia, Doutora PPCS/UNB, E-mail: silvanasf@unb.br.

${ }^{5}$ Professora Adjunta do curso de Enfermagem da Universidade de Brasília/Campus Ceilândia UNB/FCE , Doutora PIE/USP, E-mail: taniarehem@unb.br.

6 Professora Adjunta do curso de Enfermagem da Universidade de Brasília/Campus Ceilândia, Doutora PPGCS/UFG, E-mail: walterlania@unb.br.

7 Professora Adjunta do curso de Enfermagem da Universidade de Brasília/Campus Ceilândia, Doutora PPGEnf/UNB, E-mail: crgrou@unb.br.

8 Professora Adjunta do curso de Enfermagem da Universidade de Brasília/Campus Ceilândia, Doutora PPCTS/UNB, Mestre EEUFMG, E-mail: marinamorato@unb.br.

9 Professora Adjunta do curso de Enfermagem da Universidade de Brasília/Campus Ceilândia, Doutora FMRP/USP, E-mail: mani@unb.br.
} 
clinical partner, to evaluate chronic pain (Visual Analogue Scale / EVA and body diagram) and another related to daily damages. Results: The majority $(67.5 \%)$ were females with a mean age of 68.9 years and the pain was characterized as intense. The main damages were in the Daily Life Activities (ADL) and in the relationship with other people $(p=\leq 0.02)$ associated with greater pain intensity. The main site of referred pain was in the trunk and affects $39.9 \%$ at night. Conclusion: The main damages associated with pain were in the ADL and in the relationship with other people. The pain that affects the elderly is characterized as intense and appears in the evening.

Keywords: Aged. Chronic Pain. Nursing Assessment. Health services for aged. Pain Measurement. Care. Primary nursing.

\section{INTRODUÇÃO}

No Brasil, segundo o último Censo do IBGE em 2010, evidenciou-se um aumento do número do idosos entre 1999 e 2009, (de 6,4 milhões para 9,7 milhões). Neste processo surgem problemas associados ao envelhecimento, entre eles a dor crônica relacionada as doenças crônicas não Transmissíveis (DCNT), tais como obesidade e diabetes mellitus (NICHOLAS et al., 2017; IBGE, 2013). Destaca-se que a dor crônica é identificada pela Association for the Study of Pain (IASP), com prevalência média de 35,5\% (MATOS; BERNARDES; GOUBERT, 2016). Estima-se que $80 \%$ a $85 \%$ dos indivíduos idosos apresentam, pelo menos um problema significativo de saúde e predispõe a apresentar dor. Aproximadamente $50 \%$ a $60 \%$ dos pacientes tornam-se parcial ou totalmente incapacitados, transitória ou permanentemente (DRAGIOTI et al., 2016). Entre alguns prejuízos associados a dor evidencia-se as mudanças no padrão de sono, no apetite, lazer, e alteração na qualidade de vida (DELLAROZA; PIMENTA, 2012; REIS; TORRES, 2011).

As DCNT estão associadas a presença de dor e trazem prejuízos como alterações na locomoção de idosos e risco de queda (KAYSER et al., 2014; RODRIGUES et al., 2016; PIMENTA et al., 2015; CASTRO et al., 2016; LOUREÇO et al., 2013). Um estudo realizado no Paraná com 451 idosos, identificou a prevalência de dor crônica de 51,44\% (DELLAROZA et al., 2007). A dor crônica na vida diária de idosos prejudica de algum modo a realização das atividades de vida diária (AVD), bem como restringi a convivência social (isolamento) (CELICH; GALON, 2009; PAGOTTO et al., 2016; PEREIRA et al., 2014). Assim, outros estudos vêm sendo desenvolvidos para aliviar, tratar e qualificar essa dor (LOBO; SANTOS; GOMES, 2014; MANSANO-SCHLOSSER et al., 2014; PEREIRA et al., 2015; SILVA et al., 2014).

Observa-se que problemas dos idosos devido as DCNT já foi objeto de alguns estudos com avaliação da incapacidade em estudos nacionais (DRAGIOTI et al., 2016; CASTRO et al., 2016; PAGOTTO et al., 2016) e internacionais (NICHOLAS et al., 2017; DRAGIOTI et al., 2016; MATOS; BERNARDES; GOUBERT, 2016), sono, apetite, lazer (DELLAROZA; PIMENTA, 2012; REIS; TORRES, 2011), convivência social (isolamento) CELICH; GALON, 2009; PAGOTTO et al., 2016) e na qualidade de vida (DELLAROZA; PIMENTA, 2012; LOBO; SANTOS; GOMES, 2014; MANSANO-SCHLOSSER et al., 2014). 
Entretanto, estas variáveis ainda não foram associadas a dor como este estudo pretende analisá-las. Por outro lado o risco de queda já foi associado a dor em idosos (KAYSER et al., 2014; RODRIGUES et al., 2016; PIMENTA et al., 2015). Isto posto o objetivo deste estudo foi de analisar os principais prejuízos associados a dor crônica de um grupo de idosos.

\section{MÉTODO}

Estudo transversal, descritivo de abordagem quantitativa. A coleta de dados ocorreu de janeiro a julho de 2016 em uma Unidade de Saúde do Distrito Federal (cadastrados na Estratégia Saúde da Família).

A população do estudo foi constituída de idosos moradores de Ceilândia, a amostra foi por conveniência e elegeu-se uma amostra final de 268 idosos. Foram incluídos idosos: com idade mínima de 60 anos; que referiram dor persistente há mais de três meses; residente de Ceilândia e que aceitaram participar da pesquisa. Foram excluídos: idosos com deficiências cognitivas incapacitando-o de participar da pesquisa, avaliado pela Escala Pfeffer.

A coleta de dados foi realizada por meio de entrevista com os idosos para aplicação do instrumento e caracterização dados sócio demográfico, perfil clinico e as repercussões decorrentes da presença da dor crônica e seus prejuízos no cotidiano de idosos. A dor foi avaliada com uso da Escala Visual Analógica (EVA), de 0 a 10 pontos. Foi considerada nesse trabalho dor crônica como aquela presente igual ou superior a 3 meses. Posteriormente, os dados foram associados ao estado de saúde e prejuízos causados pela dor crônica no cotidiano de idosos.

Os dados foram analisados pelo software Package for the Social Sciences (SPSS $\circledast$ ) versão 21.0 para apresentação dos resultados. A análise descritiva foi realizada por frequências absoluta, relativa, medidas descritivas e de dispersão. Para comparação de médias utilizou-se o teste $t$-student e ANOVA. O nível de significância utilizado foi de $p<0,05$.

Este estudo foi aprovado pelo Comitê de Ética em Pesquisa da Secretaria do Estado de Saúde do Distrito Federal (SES/DF) № 1355211/15 e respeitou a Resolução CNS 466/2012.

\section{RESULTADOS}

Foram analisados 268 idosos que referiram dor crônica, sendo que $67,5 \%$ a maioria foi do sexo feminino. Em comparação da dor entre o sexo, as mulheres sentiram mais dor e foi caracteriza por dor intensa $(M=7,08)$. Os idosos tinham idade média de 68,9 anos (DP $\pm 6,29$, Mín.=60, Máx.=93 anos), sendo a faixa etária mais prevalente foi entre 60 e 65 
anos $(38,0 \%)$ e referiram também maior intensidade de dor como intensa $(M \geq 7,1)$ junto com a faixa etária de 81-85 anos (Tabela).

Tabela 1. Dados sociodemográficos de idosos com dor crônica de uma região administrativa do Distrito Federal, janeiro a julho de $2016(n=268)$.

\begin{tabular}{|c|c|c|c|c|c|c|c|}
\hline Variável & $\mathbf{n}$ & $\%$ & Média & DP & Mín. & Máx. & $p$ \\
\hline Sexo & & & & & & & 0,154 \\
\hline feminino & 181 & 67,5 & 7,08 & 2,523 & 1 & 10 & \\
\hline masculino & 87 & 32,5 & 6,55 & 2,74 & 1 & 10 & \\
\hline Idade & & & & & & & 0,664 \\
\hline 60 a 65 anos & 102 & 38,0 & 7,15 & 2,491 & 1 & 10 & \\
\hline 66 a 70 anos & 71 & 26,5 & 6,87 & 2,709 & 1 & 10 & \\
\hline 71 a 75 anos & 45 & 16,8 & 6,89 & 2,622 & 1 & 10 & \\
\hline 76 a 80 anos & 35 & 13,0 & 6,29 & 2,729 & 1 & 10 & \\
\hline 81 a 85 anos & 6 & 2,2 & 7,83 & 2,483 & 3 & 10 & \\
\hline 86 a 90 anos & 8 & 3,0 & 6,25 & 2,712 & 2 & 10 & \\
\hline maior que 90 anos & 1 & 4 & 8 & - & 8 & 8 & \\
\hline Escolaridade & & & & & & & 0,851 \\
\hline Nunca foi a escola & 85 & 31,7 & 6,72 & 2,759 & 1 & 10 & \\
\hline 1 a 4 serie & 143 & 53,4 & 6,94 & 2,52 & 1 & 10 & \\
\hline 5 a 8 serie & 21 & 7,8 & 7,24 & 2,644 & 2 & 10 & \\
\hline 1 a 2 serie/colegial & 10 & 3,7 & 7,1 & 2,685 & 3 & 10 & \\
\hline 3 serie/colegial & 8 & 3,0 & 7,5 & 2,673 & 3 & 10 & \\
\hline Superior completo & 1 & ,4 & 5 & - & 5 & 5 & \\
\hline Cor & & & & & & & 0,334 \\
\hline Branco & 140 & 52,2 & 7,08 & 2,584 & 1 & 10 & \\
\hline Pardo & 73 & 27,2 & 7 & 2,577 & 2 & 10 & \\
\hline Preta & 53 & 19,8 & 6,38 & 2,705 & 1 & 10 & \\
\hline Amarelo & 2 &, 7 & 6 & 0 & 6 & 6 & \\
\hline Estado Civil & & & & & & & 0,739 \\
\hline Casado/amigado & 147 & 54,9 & 7,01 & 2,588 & 1 & 10 & \\
\hline Solteiro & 22 & 8,2 & 6,55 & 2,464 & 1 & 10 & \\
\hline Viúvo & 81 & 30,2 & 6,89 & 2,716 & 1 & 10 & \\
\hline Divorciado & 18 & 6,7 & 6,61 & 2,5 & 2 & 10 & \\
\hline É aposentado & & & & & & & 0,120 \\
\hline $\operatorname{sim}$ & 105 & 39,2 & 6,59 & 2,699 & 1 & 10 & \\
\hline não & 163 & 60,8 & 7,12 & 2,525 & 1 & 10 & \\
\hline Trabalha atualmente & & & & & & & 0,299 \\
\hline $\operatorname{sim}$ & 113 & 42,2 & 7,05 & 2,695 & 1 & 10 & \\
\hline não & 155 & 57,8 & 6,81 & 2,536 & 1 & 10 & \\
\hline Renda & & & & & & & 0,823 \\
\hline$\leq 1 \mathrm{SM}$ & 187 & 69,80 & 6,74 & 2,718 & 1 & 10 & \\
\hline$\geq 1$ a $2 S M$ & 67 & 25 & 7 & 2,629 & 2 & 10 & \\
\hline$>2 \mathrm{SM}$ & 14 & 5,20 & 7,14 & 1,956 & 5 & 10 & \\
\hline
\end{tabular}

Legenda: DP (Desvio Padrão); Mín.(Mínimo); Máx. (Máximo); SM (Salário Mínimo)

A dor não foi associada aos dados sociodemográficos, contudo a faixa etária de 60 a 65 anos, que estudou $5^{\mathrm{a}}$ a $3^{\circ}$ ano colegial, de cor branco, casados, os não aposentados, que trabalham ainda e com renda superior a um SM referiu maior intensidade de dor (Intensa) (Tabela 1).

Referente as características clínicas e de saúde dos idosos, os idosos com IMC (peso normal) e que possuem Hipertensão Arterial Sistêmica (HAS) referiram a dor como intensa $(M \geq 7,0)$. Enfatiza-se que dor foi associada aos idosos que recebem algum tipo de auxilio e/ou possuem alguma dificuldade para movimentar-se referiam maior intensidade 
de dor classificada por intensa $(p \leq 0,024)$ (Tabela 2$)$.

Tabela 2. Características de saúde de idosos com dor crônica de uma região administrativa do Distrito Federal, janeiro a julho de $2016(\mathrm{n}=268)$.

\begin{tabular}{|c|c|c|c|c|c|c|c|}
\hline Variável & $\mathbf{n}$ & $\%$ & Média & DP & Mín. & Máx. & p \\
\hline IMC & & & & & & & 0,994 \\
\hline Abaixo peso $(17-18,49)$ & 9 & 3,4 & 6,56 & 2,833 & 2 & 10 & \\
\hline Peso normal $(18,5-24,9)$ & 93 & 34,7 & 7,04 & 2,532 & 2 & 10 & \\
\hline Acima do peso $(25-29,9)$ & 100 & 37,3 & 6,88 & 2,587 & 1 & 10 & \\
\hline Obesidade I $(30-34,9)$ & 49 & 18,3 & 6,82 & 2,766 & 1 & 10 & \\
\hline Obesidade II $(35-39,9)$ & 13 & 4,9 & 6,85 & 2,882 & 1 & 10 & \\
\hline Obesidade III (>40) & 4 & 1,5 & 6,75 & 2,63 & 4 & 9 & \\
\hline $\begin{array}{l}\text { Pratica alguma atividade } \\
\text { física diária }\end{array}$ & & & & & & & 0,664 \\
\hline Sim & 102 & 38,1 & 6,96 & 2,681 & 1 & 10 & \\
\hline Não & 166 & 61,9 & 6,88 & 2,56 & 1 & 10 & \\
\hline $\begin{array}{l}\text { Internação nos últimos } 6 \\
\text { meses }\end{array}$ & & & & & & & 0,653 \\
\hline Sim & 53 & 19,8 & 6,98 & 2,811 & 1 & 10 & \\
\hline Não & 215 & 80,2 & 6,89 & 2,555 & 1 & 10 & \\
\hline $\begin{array}{l}\text { Necessita de algum tipo d } \\
\text { auxílio }\end{array}$ & & & & & & & 0,024 \\
\hline Sim & 72 & 26,9 & 7,56 & 2,22 & 2 & 10 & \\
\hline Não & 196 & 73,1 & 6,67 & 2,696 & 1 & 10 & \\
\hline $\begin{array}{l}\text { Tem dificuldade para } \\
\text { movimentar }\end{array}$ & & & & & & & 0,002 \\
\hline Sim & 132 & 49,2 & 7,4 & 2,474 & 1 & 10 & \\
\hline Não & 136 & 50,8 & 6,44 & 2,652 & 1 & 10 & \\
\hline HAS & & & & & & & 0,244 \\
\hline Sim & 194 & 72,4 & 7,02 & 2,593 & 1 & 10 & \\
\hline Não & 74 & 27,6 & 6,62 & 2,621 & 1 & 10 & \\
\hline DM & & & & & & & 0,862 \\
\hline Sim & 61 & 22,8 & 6,87 & 2,884 & 1 & 10 & \\
\hline Não & 207 & 77,2 & 6,92 & 2,521 & 1 & 10 & \\
\hline
\end{tabular}

Legenda: DP (Desvio Padrão); HAS (Hipertensão Arterial Sistêmica); DM (Diabetes Mellitus);

Mín.(Mínimo); Máx. (Máximo); SM (Salário Mínimo)

A maioria dos idosos não se internou nos últimos seis meses e caracterizaram a sua dor como moderada $(M \geq 6,8)$ junto com que possuem $D M$.

A mensuração da intensidade geral de dor da dor pela escala EVA foi que a maioria (37\%) dos idosos a classificou como dor intensa $(M=8,6, D P= \pm 0,74$, Máx.=9,0, Mín.=7,0), $28 \%$ dor moderada $(M=5,15, D P= \pm 0,73$, Máx. $=6,0$, Mín. $=4,0), 22 \%$ referiram como a pior dor possível (M=10,0, DP=t---, Máx.=10,0, Mín.=10,0) e por fim 13\% descreveu como uma dor leve $(M=2,2, D P= \pm 0,75$, Máx.=3,0, Mín.=1,0).

Observou-se que 39,9\% referiram que a dor aparece no período da noite. Em relação ao prejuízo que a dor acarreta em seu dia, os prejuízos das AVD e na relação com outras pessoas os idosos referiram maior intensidade de dor (intensa) $(p \leq 0,000)$. Contudo o prejuízo na atividade social não foi associado a dor ( $p \geq 0,109)$, mas também referiam como intensa (Tabela 3).

Já referente ao tratamento utilizado para a dor que sentem, a maioria utiliza antiinflamatórios, opióides e os não usam outros tipos medicamentos e esses classificaram a 
dor como moderada $(M=\geq 6,8)$. Porém a dor foi associada $(p \leq 0,016)$ ao não uso de analgésicos $(42,5 \%)$, e a referiram maior intensidade de dor (intensa).

Tabela 3. Características da dor e prejuízos de idosos com dor crônica de uma região administrativa do Distrito Federal, janeiro a julho de $2016(n=268)$.

\begin{tabular}{|c|c|c|c|c|c|c|c|}
\hline Variável & $\mathbf{n}$ & $\%$ & Média & DP. & Mín. & Máx. & $\mathbf{p}$ \\
\hline Dor aparece & & & & & & & 0,865 \\
\hline manhã & 79 & 29,5 & 6,81 & 2,592 & 1 & 10 & \\
\hline tarde & 82 & 30,6 & 6,98 & 2,802 & 1 & 10 & \\
\hline noite & 107 & 39,9 & 6,93 & 2,47 & 1 & 10 & \\
\hline Tempo que sente dor & & & & & & & 0,019 \\
\hline$<3$ meses & 25 & 9,3 & 5,48 & 2,917 & 1 & 10 & \\
\hline 3 a 6 meses & 9 & 3,4 & 5,89 & 2,619 & 2 & 10 & \\
\hline$>6$ meses a 1 ano & 24 & 9 & 6,46 & 2,553 & 2 & 10 & \\
\hline$>1$ a 5 anos & 66 & 24,6 & 6,8 & 2,451 & 1 & 10 & \\
\hline$>5$ a a10 anos & 49 & 18,3 & 7,33 & 2,585 & 1 & 10 & \\
\hline$>10$ anos & 95 & 35,4 & 7,36 & 2,513 & 1 & 10 & \\
\hline Prejuízo das atividades sociais & & & & & & & 0,109 \\
\hline $\operatorname{sim}$ & 65 & 24,3 & 7,37 & 2,485 & 1 & 10 & \\
\hline não & 203 & 75,7 & 6,76 & 2,628 & 1 & 10 & \\
\hline Prejuízo das AVD & & & & & & & 0,000 \\
\hline $\operatorname{sim}$ & 150 & 56 & 7,49 & 2,376 & 1 & 10 & \\
\hline não & 118 & 44 & 6,18 & 2,701 & 1 & 10 & \\
\hline $\begin{array}{l}\text { Prejuízo na relação com } \\
\text { outras pessoas }\end{array}$ & & & & & & & 0,000 \\
\hline Sim & 41 & 15,3 & 8,35 & 2,02 & 1 & 10 & \\
\hline Não & 227 & 84,7 & 6,64 & 2,61 & 1 & 10 & \\
\hline $\begin{array}{l}\text { Tratamento com Anti- } \\
\text { inflamatórios }\end{array}$ & & & & & & & 0,493 \\
\hline Sim & 117 & 43,7 & 7,01 & 2,731 & 1 & 10 & \\
\hline Não & 151 & 56,3 & 6,86 & 2,548 & 1 & 10 & \\
\hline Tratamento com Analgésicos & & & & & & & 0,016 \\
\hline Sim & 154 & 57,5 & 6,59 & 2,655 & 1 & 10 & \\
\hline Não & 114 & 42,5 & 7,35 & 2,528 & 1 & 10 & \\
\hline Tratamento com Opioides & & & & & & & 0,171 \\
\hline Sim & 20 & 7,5 & 7,77 & 2,743 & 1 & 10 & \\
\hline Não & 248 & 92,5 & 6,88 & 2,614 & 1 & 10 & \\
\hline $\begin{array}{l}\text { Tratamento com outros } \\
\text { medicamentos }\end{array}$ & & & & & & & 0,243 \\
\hline Sim & 37 & 13,8 & 6,47 & 2,543 & 1 & 10 & \\
\hline Não & 231 & 86,2 & 6,98 & 2,633 & 1 & 10 & \\
\hline
\end{tabular}

Legenda: DP (Desvio Padrão); HAS (Hipertensão Arterial Sistêmica); DM

(Diabetes Mellitus) Mín.(Mínimo); Máx. (Máximo); SM (Salário Mínimo) 
O gráfico 1 ilustra os principais locais que os idosos sentem dor associado a média do nível de dor (representada pela linha, 0-10 pontos EVA) que sentem em cada local. Pode-se verificar a dor no tronco e nos membros inferiores (MMII), representou os principais locais de dor, descrita por dor moderada. Contudo o local de menor frequência de dor, dor de cabeça, foi o local onde referiam pior dor, intensa $(M=7,7)(p \leq 0,01)$ (Gráfico 1).

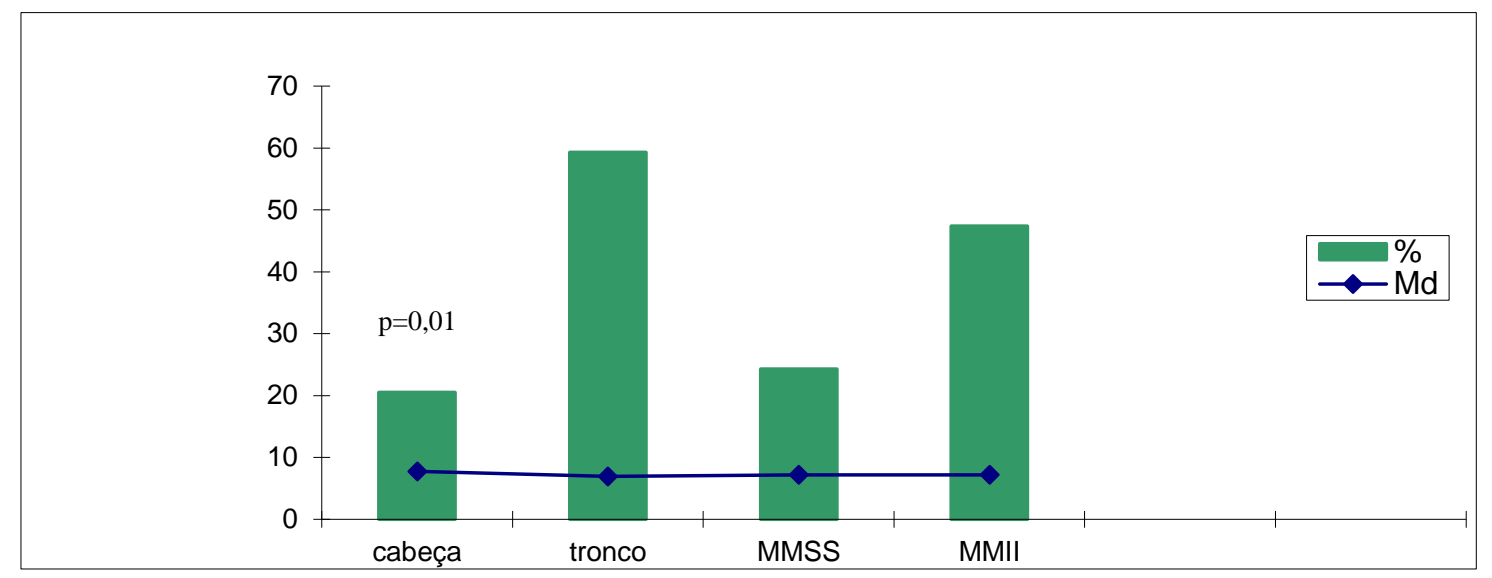

Gráfico 01. Localização e intensidade de dor (escala numérica/média), de idosos com dor crônica de uma região administrativa do Distrito Federal, janeiro a julho de $2016(n=268)$.

\section{DISCUSSÃO}

Como foi encontrado nessa pesquisa, o sexo feminino foi o mais prevalente, e isso ocorre na maioria dos estudos sobre idosos e/ou com dor crônica em estudos nacionais (REIS; TORRES, 2011; RODRIGUES et al., 2016; CELICH; GALON, 2009; DELLAROZA et al., 2008; KREBS et al., 2016; TRELHA et al., 2006) e estudos internacionais (SÁ et al., 2009; NICHOLAS et al., 2017). A média de idade encontrada foi menor que de outros estudos (REIS; TORRES, 2011; CORRÊA; CEOLIM, 2008; TRELHA et al., 2006), porém a maior prevalência de 60-65 anos está de acordo com várias outras pesquisas (DELLAROZA; PIMENTA, 2012; REIS; TORRES, 2011; DELLAROZA et al., 2007; KREBS et al., 2016), podendo a variação chegar até 75 anos, sendo que os estudos internacionais apresentam uma idade superior a 65 anos(NICHOLAS et al., 2017; MATOS; BERNARDES; GOUBERT, 2016; KREBS et al., 2016)

Aqueles que não praticam atividade física e não fumam, também são maioria, assim como nesse estudo, em outra pesquisa da população de Salvador (PAGOTTO et al., 2016), demonstrou que $71,7 \%$ e $58,9 \%$, respectivamente, levam o mesmo estilo de vida, mas vale ressaltar que outra pesquisa apontou que os fumantes ou ex-fumantes são mais vulneráveis à desenvolverem uma dor crônica (SÁ et al., 2009). Estudos internacionais têm evidenciado que a presença de dor crônica tem limitado as pessoas a realizarem atividade física (MATOS; BERNARDES; GOUBERT, 2016; KREBS et al., 2016; CROWE et al., 2017; LARSSON et al., 2016).

Apesar de que os que estão acima do peso, IMC entre 25-29,9 serem os mais 
prevalentes, a dor referida demonstrou um nível moderado. Sabe-se que obesidade é um fator de risco para ocasionar a dor crônica, pois ela pode determinar várias morbidades associada ao desenvolvimento de complicações que desencadeia a presença de ocorrência da dor crônica associadas aos distúrbios osteomusculares (PEREIRA et al., 2014; PEREIRA et al., 2015; OLIVEIRA; VENCIO, 2016).

Em relação às condições de saúde da amostra dessa pesquisa, a maioria dos idosos possuíam HAS o que reflete a realidade da população brasileira, pois o número de desenvolvimento de doenças cardiovasculares vem progredindo. No estudo com 48 idosos Cruzaltense, 70,4\% dos idosos possuíam o diagnóstico de HAS (CELICH; GALON, 2009) e em outro estudo de caracterização de 24 idosos restritos ao seu domicílio e seus cuidadores no Paraná, $70,8 \%$ tinham HAS, ressaltando o resultado com proximidade dos valores obtido nessa pesquisa (TRELHA et al., 2006).

A dor teve associação com aqueles idosos que precisam de algum auxílio e/ou possui algum tipo de dificuldade para movimentar-se. Pode-se assim, relacionar essa diminuição da capacidade de deambular com o local da dor, pois conforme o gráfico 1, 0 segundo principal local foram nos MMII, o que dificulta o caminhar e aumenta a dependência de auxilio nesses idosos, sendo que a dor nos membros inferiores é bastante prevalente em diversos estudos nacionais (DELLAROZA; PIMENTA, 2012; REIS; TORRES, 2011; PIMENTA et al., 2015

CASTRO et al., 2016; CELICH; GALON, 2009; PEREIRA et al., 2015). Nos estudos internacionais os idosos com problemas de coluna, incapacidades, idosos com histórico de quedas (MATOS; BERNARDES; GOUBERT, 2016; KREBS et al., 2016; SCHERER et al., 2016; CROWE et al., 2017).

Referente a dor e prejuízos, o período do dia que a dor mais acometeu os idosos foi durante a noite. O mesmo resultado foi encontrado em outra pesquisa, no qual $44,5 \%$ dos idosos pesquisados também referiram que o surgimento da dor acontecia durante a noite (CELICH; GALON, 2009). Também outro estudo pouco mais da metade da amostra não tinha horário preferencial para aparecer a dor, porém dentre os que tinham $24,7 \%$ referiram surgir no período noturno (DELLAROZA et al., 2008). Outros pesquisadores também afirmam que o aparecimento dor esta geralmente relacionado com o executar de alguma atividade (levantar, andar, sentar, etc.) (PIMENTA et al., 2015; CASTRO et al., 2016; SCHERER et al., 2016).

Observa-se que a presença de dor em idosos pelo período noturno podem ser associadas as complicações de DCNT como a evolução de DM após os primeiros 10 anos de patologia. Esta dor pode evoluir para neuropatia diabética (PEREIRA et al., 2015; OLIVEIRA; VENCIO, 2016). Desta forma a enfermagem e a equipe de saúde devem estar atentas na investigação ativa dos idosos para prevenção de dores neuropáticas associadas a diabetes. Em estudo internacionais a neuropatia tem acometidos os idosos e traz prejuízos em seu cotidiano (KREBS et al., 2016; SCHERER et al., 2016; LARSSON et al., 2016) 
A dor foi associada também ao tempo em que os idosos sentem essa dor, sendo que a maioria refere senti-la a mais de 10 anos e a intensidade de dor é classificada como intensa entre os que a sentem a mais de 5 anos. Por diferenças metodológicas, poucos são os estudos que trazem o tempo que os idosos manifestam dor crônica (LOBO; SANTOS; GOMES, 2014; PEREIRA et al., 2015; SCHERER et al., 2016), apenas um estudo (DELLAROZA et al., 2007) teve como resultado de sua amostra que $51,4 \%$ dos idosos referiram dor com duração igual ou superior que 6 meses. As pesquisas internacionais também tem considerado um tempo superior a seis meses (MATOS; BERNARDES; GOUBERT, 2016; KREBS et al., 2016; LARSSON et al., 2016).

A frequência dos episódios de dor é fator estudado por alguns autores em seus estudos sobre a prevalência da dor crônica, sendo que a maioria dos idosos queixa-se que a dor se manifesta diariamente, e em segundo lugar vem o aparecimento em frequência variável(DELLAROZA; PIMENTA, 2012; DELLAROZA et al., 2008).

No contexto social as pessoas, e incluindo os idosos, que sentem algum tipo de dor começam imediatamente a procurar ou tomar algum tipo de medicamento para o alívio da dor (PEREIRA et al., 2015). Os idosos investigados em outro estudo (CELICH; GALON, 2009), 96,3\% admitiram tomar alguma medida de alívio à dor, sendo que 33,3\% assumiram ser tratamento farmacológico com algum medicamento e $29,7 \%$ realizam massagem com pomadas, remédios caseiros. Ressalta que apesar dos idosos com dor crônica não utilizarem as medidas não farmacológicas elas são importantes para diminuir a necessidade do uso de medicamentos (PEREIRA et al., 2015; SÁ et al., 2009). Nos estudos internacionais também estimulam a independência com menor uso de medicamentos principalmente o de opioides que podem interferir em algumas atividades cotidianas (KREBS et al., 2016; CROWE et al., 2017).

Uma dor mal controlada pode ter consequências importantes na vida e rotina dos idosos com dor crônica, pois eles vão começar a evitar as atividades que desencadeiam essa dor e se forem ações como andar, levantar, entre outras realizadas rotineiramente, eles terão dificuldades de deambulação, ficarão mais dependentes nas AVDs e reduzirão em nível considerável sua qualidade de vida (REIS; TORRES, 2011). A qualidade de vida nos estudos internacionais tem sido identificada como pior nos idosos que tem dor crônica com maior intensidade (NICHOLAS et al., 2017; SCHERER et al., 2016).

As principais interferências da dor crônica nos idosos foram investigadas em outro cenário e observaram $40 \%$ afeta o sono, 39,0\% humor, 36,7\% lazer e 20,9\% no apetite, sendo que essa interferência foi avaliada de moderada à intensa em $50 \%$ dos casos ${ }^{(6)}$. Ainda os resultados da pesquisa realizado em Londrina (REIS; TORRES, 2011) quando comparado e relacionado com os achados deste estudo, pode-se relacionar que prejuízos no humor e no lazer prejudicam o relacionamento interpessoal dos idosos com outras pessoas, assim como prejuízos no sono e no apetite geram prejuízos físicos aos idosos (maior fadiga) o que acarreta na interferência nas AVDs dos idosos. As pesquisas internacionais tem identificado que há redução das AVD associada a presença de dor crônica (KREBS et al., 2016; LARSSON et al., 2016) e por outro lado a atividade física tem 
ajudado a melhorar os prejuízos associado a dor crônica (JACKSON et al., 2016; LARSSON et al., 2016).

Outro estudo analisou identificou que $81,5 \%$ dos idosos com dor crônica interfere de algum modo no cotidiano, na rotina e nas atividades realizadas. As principais AVDs encontradas que sofrem alguma interferência são transporte/locomoção, vestuário, trabalhar e higiene pessoal, sendo que alguns idosos têm mais que um prejuízo (CELICH; GALON, 2009).

Referente a intensidade de dor, a maioria dos idosos analisados referiram o nível de dor como intensa. Em estudo similar, com 451 idosos servidores municipais de Londrina, a maioria das queixas de dor teve sua intensidade variando de moderada à intensa (DELLAROZA; PIMENTA, 2012). Resultados semelhantes também ocorreram em estudos com idosos institucionalizados, como o realizado com 60 idosos de uma instituição de acolhimento na BA, sendo que $51,7 \%$ dos idosos declararam sentir uma dor intensa6 (REIS; TORRES, 2011). Por estes resultados, pode-se verificar o quanto a dor intensa pode ser grave tanto em aspectos físicos, como por exemplo, uma maior incapacidade nas AVDs, quanto em consequências mentais (baixa autoestima, depressão, ansiedade, etc.), fato observado em estudos nacionais DELLAROZA; PIMENTA, 2012; RODRIGUES et al., 2016) e internacionais (NICHOLAS et al., 2017; LARSSON et al., 2016).

Em relação aos locais acometidos pela dor, a maior prevalência de queixa de dor foi no tronco e nos Membros Inferiores (MMII). Cabe ressaltar que o tronco nesse estudo inclui dores na coluna. O estudo com 48 idosos moradores da cidade de Cruzaltense obteve resultados semelhantes, 44,4\% relataram dor na coluna lombar, seguido de $40,7 \%$ e $25,9 \%$ queixando de dor, respectivamente, nas pernas e articulação do joelho, caracterizando como MMII (CELICH; GALON, 2009). Já em outra pesquisa a coluna foi o segundo lugar com mais relatos de dor (25,0\%), ficando atrás apenas dos MMII $(47,7 \%)$, porém mantendo os principais locais de dor que são os membros inferiores e a coluna (RODRIGUES et al., 2016; STORCHI et al., 2016). Em outros países também os achados são similares para região lombar como região prevalente (JACKSON et al., 2016: SCHERER et al., 2016).

Apesar da localização na cabeça ser com menor incidência de queixa de dor, ela foi classificada como a de maior prejuízo descrita como dor intensa e o tronco teve a maior incidência descrito como dor moderada. Um estudo identificou a dor na região dorsal (leve) em $50 \%$ dos casos e moderada em $42,7 \%$, o que um pouco se assemelha à esse estudo na qual a média de dor descrita pelos idosos foi classificada como moderada, já nos MMII, a dor foi descrita como leve em $53,6 \%$ dos casos, moderada em 35\% e 11,3\% como intensa, o que já entra em contradição com esse estudo, pois a média de dor das queixas dos idosos analisados foi classificada como intensa nos MMII (CELICH; GALON, 2009).

Apesar de ser o sintoma neurológico mais comum, ocorre uma diminuição da prevalência da cefaleia nos idosos, pois ela diminui em relação às outras faixas etárias. Ainda que existam diversas classificações da cefaleia e de suas manifestações, a grande maioria delas são mais prevalentes nos jovens e sua intensidade tende a ser também maior nas faixas etárias menores (PEREIRA et al., 2015). Tais dados de baixa incidência de dor 
na cabeça dentre os idosos coincidem com resultado dessa pesquisa, porém o mesmo não ocorre em relação à intensidade dessa dor, já que nesse estudo a maior média de dor foi da dor localizada na cabeça.

Observa-se que o enfermeiro deve estar atento a identificar as causas de cefaleia em idosos, com vistas a afastar/reconhecer a cefaleia como sintoma de complicações de doenças como crises hipertensivas e Acidente Vascular Cerebral como um fator de risco associado as cefaleias em idosos.

\section{CONCLUSÃO}

Neste estudo a dor crônica esteve presente em idosos do sexo feminino, descrita como dor intensa e que aparece no período da noite. Os idosos que sente dor superior a 10 anos apresentaram com maior nível de intensidade de dor. Os principais prejuízos foram associados aos prejuízos das AVD e na relação com outras pessoas.

Os prejuízos da dor crônica reflete na vida dos idosos que podem acarretar em déficits no dia a dia deles. Esses prejuízos vão além dos aspectos físicos e se estendem para o psicológico e o social e por isso acarretam em várias consequências na autoestima, na dependência, no isolamento e relações e na qualidade de vida dessa população. Vários são os fatores que interferem e agravam esses prejuízos, por exemplo, o local, a intensidade da dor, a dependência para locomoção, a quanto tempo sentem essa dor, o tratamento utilizado, entre outros.

O enfermeiro como integrante da equipe de saúde em suas diversas áreas de conhecimento deve então se atentar e se qualificarem para a alta prevalência dessa dor crônica nos idosos e assim realizar o adequado manejo, tratamento e controle dela, com o objetivo de reduzir os prejuízos encontrados e aumentar a qualidade de vida que a população idosa tanto necessita para o seu dia a dia.

\section{AGRADECIMENTOS}

Pesquisa Financiado pelo Conselho Nacional de Pesquisa CNPQ.

\section{REFERÊNCIAS}

CASTRO, D. C., et al. Incapacidade funcional para atividades básicas de vida diária de idosos: estudo populacional/Functional disability for basic activities of daily lives of the elderly: a population study. Cienc Cuid Saude v.15, n.1, p.109-117. 2016.

CELICH, K. L. S.; GALON, C. Dor crônica em idosos e sua influência nas atividades da vida diária e convivência social. Rev Bras. Geriatr. Gerontol. v.12, n.3, p.345-359. 2009. 
CORRÊA, K.; CEOLIM, M. F. Qualidade do sono em pacientes idosos com patologias vasculares periféricas. Rev. Esc. Enferm. USP. v.42, n.1, p.12-8. 2008.

CROWE, M., et al. Older peoples' strategies for coping with chronic non-malignant pain: A qualitative meta-synthesis. Int J Nurs Stud. v.68, n.3, p.40-50. 2017.

DELLAROZA, M. S. G., et al. Caracterização da dor crônica e métodos analgésicos utilizados por idosos da comunidade. Rev Assoc. Med. Bras. n.54, n.1, p.36-41. 2008.

DELLAROZA, M. S. G.; PIMENTA, C. A. M. Impacto da dor crônica nas atividades de vida diária de idosos da comunidade. Ciênc. cuid. Saúde. v. 11, Sup. 1, p.235-242. 2012.

DELLAROZA, M. S. G.; PIMENTA, C. A. M.; MATSUO, T. Prevalência e caracterização da dor crônica em idosos não institucionalizados. Cad. Saúde Pública. v.23, n.5, p.11511160. 2007.

DRAGIOTI, E., et al. Distinct subgroups derived by cluster analysis based on pain characteristics and anxiety-depression symptoms in Swedish older adults with chronic pain. European Psychiatry. v.33, Sup.1, p.24-25, 2016.

INSTITUTO BRASILEIRO DE GEOGRAFIA E ESTATÍSTICA-IBGE. Brasil em números. Rio de Janeiro, v.21, p.1-392. 2013 Disponível em:

http://biblioteca.ibge.gov.br/visualizacao/periodicos/2/bn_2013_v21.pdf. Acesso em: 15 fev 2016.

JACKSON T, et al. Systematic Review and Meta-Analysis of the Global Burden of Chronic Pain Without Clear Etiology in Low- and Middle-Income Countries: Trends in Heterogeneous Data and a Proposal for New Assessment Methods. Anesth Analg. v.123, n.3, p.739-48. 2016.

KAYSER B., et al. Influência da dor crônica na capacidade funcional do idoso. Rev. Dor. v.15, n.1, p.48-50. 2014.

KREBS, E. E, et al. Association of Opioids with Falls, Fractures, and Physical Performance among Older Men with Persistent Musculoskeletal Pain. J Gen Intern Med. v.31, n.5, p.463-9. 2016.

LARSSON C., et al. Impact of pain characteristics and fear- avoidance beliefs on physical activity levels among older adults with chronic pain: a population-based, longitudinal study. BMC Geriatr. v.24, n.1, p.16-50. 2016.

LOBO, A. J. S.; SANTOS, L.; GOMES, S. Nível de dependência e qualidade de vida da população idosa. Rev Bras Enferm. v.67, n.6, p.913-9. 2014.

LOURENCO, T. S., et al. Fatores ambientais de risco para quedas em idosos moradores de Ceilândia-DF. Movimenta. v.6, n.2, p.471-480. 2013.

MANSANO-SCHLOSSER, T. C., et al. Idosos institucionalizados: organização cronológica das rotinas diárias e qualidade do sono. Rev Bras Enferm. v.67, n.4, p.610-6. 2014.

MATOS, M.; BERNARDES, S. F.; GOUBERT, L. The relationship between perceived promotion of autonomy/ dependence and pain-related disability in older adultsisepith 
chronic pain: the mediating role of self-reported physical functioning. J Behav Med. v.39, p.704-715. 2016.

NICHOLAS, M. K., et al. Long-term outcomes from training in self-management of chronic pain in an elderly population: a randomized controlled trial. Pain. v.158, n.1, p.86-95. 2017.

OLIVEIRA, J. E. P.; VENCIO, S. Diretrizes da Sociedade Brasileira de Diabetes 20152016, 2016. Disponível em:

http://www.diabetes.org.br/profissionais/images/docs/DIRETRIZES-SBD-2015-2016.pdf. Acesso em: 13 dez 2016.

PAGOTTO, V., et al. Comparação da funcionalidade de idosos residentes em duas modalidades institucionais. Revista Eletrônica de Enfermagem. v.18, n.2, p.34712. 2016.

PEREIRA, L. V., et al. Prevalence and intensity of chronic pain and self-perceived health among elderly people: a population-based study. Revista Latino-Americana de Enfermagem. v.22, n.4, p.662-669. 2014

PEREIRA, L. V., et al.Pain intensity among institutionalized elderly: a comparison between numerical scales and verbal descriptors. Rev. esc. enferm. USP. v.49, n.5, p. 804-810. 2015.

PIMENTA, W. R. T., et al. Quedas e qualidade de vida: associação com aspectos emocionais em idosos comunitários. Geriatrics, Gerontology and Aging, v.9, n.3, p.4248. 2015.

REIS, L. A.; TORRES, G. V. Influência da dor crônica na capacidade funcional de idosos institucionalizados. Rev. bras. enferm. v. 64, n. 2, p.274-280. 2011.

RODRIGUES D. et al. Prevalence of chronic pain among elderly living in a city of Northern Rio Grande do Sul. Rev. Dor. v.17, n.3, p.201-204. 2016.

SÁ, K., et al. Prevalência de dor crônica e fatores associados na população de Salvador, Bahia. Rev Saúde Pública. v.43, n.4, p.622-630. 2009.

SCHERER, M., et al. Association between multimorbidity patterns and chronic pain in elderly primary care patients: a cross-sectional observational study. BMC Fam Pract. v17, n.68, p.21-31, 2016.

SILVA, A. G., et al. Pain intensity is associated with both performance-based disability and self-reported disability in a sample of older adults attending primary health care centers.

Disabil. Health J. v.7, n.4, p.457-65. 2014.

STORCHI, S., et al. Quality of life and anxiety and depression symptoms in elderly females with and without chronic musculoskeletal pain. Rev Dor. v.17, n.4, p.283-8. 2016.

TRELHA, C. S., et al. Caracterização de idosos restritos ao domicílio e seus cuidadores. Revista Espaço para a Saúde. v.8, n.1, p.20-27. 2006. 